\title{
Integral Equations on the Half-Line: A Modified Finite-Section Approximation
}

\author{
By I. H. Sloan and A. Spence
}

\begin{abstract}
We consider the approximate solution of integral equations of the form $y(t)$ $\int_{0}^{x} k(t, s) y(s) d s=f(t)$, where the conditions on $k(t, s)$ are such that kernels of the Wiener-Hopf form $k(t, s)=\kappa(t-s)$ are included in the analysis. The finite-section approximation, in which the infinite integral is replaced by $\int_{0}^{\beta}$ for some $\beta>0$, yields an approximate solution $y_{\beta}(t)$ that is known, under very general conditions, to converge to $y(t)$ as $\beta \rightarrow \infty$ with $t$ fixed. However, the convergence is uniform only on finite intervals, and the approximation is typically very poor for $t>\beta$. Under the assumption that $f$ has a limit at infinity, we here introduce a modified finite-section approximation with improved approximation properties, and prove that the new approximate solution converges uniformly to $y$ as $\beta \rightarrow \infty$. A numerical example illustrates the improvement.
\end{abstract}

1. Introduction. We consider integral equations of the form

$$
y(t)-\int_{0}^{\infty} k(t, s) y(s) d s=f(t), \quad t \in \mathbf{R}^{+},
$$

or

$$
y-K y=f,
$$

under the assumptions that $f$ and $y$ are bounded, continuous functions on $\mathbf{R}^{+}$, and that $f$ has a limit at infinity. The conditions on $k(t, s)$, to be stated fully in Section 2, admit kernels of the Wiener-Hopf form $k(t, s)=\kappa(t-s)$, where $\kappa \in L(\mathbf{R})$. Many radiative transfer problems in astrophysics (see, for example, [4]) are of the form assumed here.

We shall assume throughout that $1 \notin \sigma(K)$, where $\sigma(K)$ is the spectrum of $K$ in the space $X^{+}$of bounded continuous functions on $\mathbf{R}^{+}$with norm

$$
\|g\|=\sup _{t \in \mathbf{R}^{+}}|g(t)| \text {. }
$$

Then (1) has a unique solution $y \in X^{+}$for each $f \in X^{+}$.

The conventional finite-section approximation is defined, for $\beta \in \mathbf{R}^{+}$, by

$$
y_{\beta}(t)-\int_{0}^{\beta} k(t, s) y_{\beta}(s) d s=f(t), \quad t \in \mathbf{R}^{+} .
$$

Recently, Anselone and Sloan [1], extending earlier work of Atkinson [2], have established that $y_{\beta}(t)$ converges to $y(t)$ as $\beta \rightarrow \infty$, and that the convergence is uniform for $t$ in any finite interval. On the other hand, the convergence is in general not uniform on $\mathbf{R}^{+}$. This behavior is illustrated by an example in Section 4 .

Received March 19, 1985; revised October 15, 1985

1980 Mathematics Subject Classification. Primary 65R20, 45L10; Secondary 45E10. 
The main purpose of this paper is to show that a uniform approximation to $y$ may be obtained by a simple modification of the finite-section approximation. The modification is based on the fact (see [7]) that $y$, like $f$, has a limit at infinity; and, moreover, the limit $y(\infty)$ is available without solving the integral equation (see Theorem 1). Using this information, we make the approximation

$$
\int_{\beta}^{\infty} k(t, s) y(s) d s \simeq \int_{\beta}^{\infty} k(t, s) y(\infty) d s
$$

and thus approximate (1) by the equation

$$
z_{\beta}(t)-\int_{0}^{\beta} k(t, s) z_{\beta}(s) d s=f_{\beta}(t)
$$

where

$$
f_{\beta}(t)=f(t)+\int_{\beta}^{\infty} k(t, s) y(\infty) d s .
$$

We call $z_{\beta}$ the modified finite-section approximation to $y$. Note that the modified equation (4) has the same form as the conventional finite-section equation (2), except that the inhomogeneous term $f$ is now replaced by a modified term $f_{\beta}$. Intuitively, it seems clear that $z_{\beta}$ will be a better approximation than $y_{\beta}$ when $t$ is large. This notion is made precise in Section 3, where we prove that $z_{\beta}$ converges uniformly to $y$ on $\mathbf{R}^{+}$as $\beta \rightarrow \infty$. In Section 4 , a numerical example illustrates the improved approximation properties of the modified finite-section approximation over the conventional approximation.

It should be noted that (2) and (4) are in effect integral equations over the finite interval $[0, \beta]$, and so may be solved by any of the standard methods for such problems, for example, by quadrature (see [2] or Section 4), collocation, or Galerkin's method. The method of collocation with piecewise-constant basis elements applied to (4) can be seen to be equivalent, at least on $[0, \beta]$, to a certain collocation method applied to the original equation (1), namely, the method of 'collocation at $\infty$ '; see [7, Section 6]. In [7] the authors discuss the stability, convergence and global superconvergence of this and other projection methods applied to (1).

2. Limits at Infinity. The kernel $k(t, s)$ in (1) will be assumed to be expressible in the form

$$
k(t, s)=\kappa(t-s)+l(t, s),
$$

where $\kappa \in L(\mathbf{R})$, and where $l$ satisfies the three conditions:

$$
\begin{aligned}
& \sup _{t} \int_{0}^{\infty}|l(t, s)| d s<\infty \\
& \int_{0}^{\infty}\left|l\left(t^{\prime}, s\right)-l(t, s)\right| d s \rightarrow 0 \quad \text { as } t^{\prime} \rightarrow t, \text { for } t \in \mathbf{R}^{+}, \\
& \int_{0}^{\infty}|l(t, s)| d s \rightarrow 0 \text { as } t \rightarrow \infty .
\end{aligned}
$$

We shall assume from now on that $f$ is in $C_{l}^{+}$, the subspace of $X^{+}$consisting of the continuous functions on $\mathbf{R}^{+}$that have a limit at infinity. In this space, the integral operator $K$ with kernel $k(t, s)$ is a bounded operator, with norm

$$
\|K\|=\sup _{t} \int_{0}^{\infty}|k(t, s)| d s .
$$

However, it is a compact operator if and only if $\kappa=0$ (see [2], [6]). 
The spectrum of $K$ in the space $C_{l}^{+}$is shown in [7] to be the same as in the space $X^{+}$, i.e., in the notation of Section 1 , to be $\sigma(K)$. Thus, for $1 \notin \sigma(K)$, Eq. (1) has a unique solution $y \in C_{l}^{+}$for each $f \in C_{l}^{+}$. The existence and uniqueness in $C_{l}^{+}$of the solutions $y_{\beta}$ and $z_{\beta}$ of (2) and (4), for $\beta$ sufficiently large, is established in the following section.

Given the existence of the limits at infinity, it is an easy matter to take the limit $t \rightarrow \infty$ in (1), (2), and (4), and so obtain the following theorem:

Theorem 1. Assume $1 \notin \sigma(K)$ and $f, y_{\beta}, z_{\beta} \in C_{l}^{+}$. Then

$$
y(\infty)=\frac{1}{1-\chi} f(\infty), \quad y_{\beta}(\infty)=f(\infty), \quad z_{\beta}(\infty)=y(\infty)
$$

where

$$
\chi=\int_{-\infty}^{\infty} \kappa(u) d u
$$

The first result is proved in [7]. The second and third results follow in a straightforward way. From [7] we also draw the remark that the denominator $1-\chi$ cannot vanish for $1 \notin \sigma(K)$.

It is immediately clear from Theorem 1 that $y_{\beta}(\infty) \neq y(\infty)$, unless either $f(\infty)=0$ or $\chi=0$. Hence (2) should not be used if an approximation to $y(t)$ is required on the whole of $[0, \infty)$. On the other hand, $z_{\beta}(t)$ has the correct limit at $\infty$, thus opening the possibility (which we shall later confirm) that it is an acceptable approximation for all $t$ in $[0, \infty)$.

3. The Modified Finite-Section Approximation. The main results in this section are that the modified finite-section approximation $z_{\beta}$, the solution of (4), exists and is unique for $\beta$ sufficiently large (Theorem 2), and converges uniformly to $y$ (Theorem 3).

To aid the discussion, it is convenient to introduce the finite-section integral operator,

$$
K_{\beta} g(t)=\int_{0}^{\beta} k(t, s) g(s) d s .
$$

Clearly, $K_{\beta}$ is a bounded operator on $C_{l}^{+}$, with $\left\|K_{\beta}\right\| \leqslant\|K\|$. The conventional finite-section approximation (2) and the modified approximation (4) may be written as

$$
y_{\beta}-K_{\beta} y_{\beta}=f
$$

and

$$
z_{\beta}-K_{\beta} z_{\beta}=f_{\beta},
$$

respectively. Thus, in both cases, the existence and uniqueness of the approximations is established if we can prove the existence of $\left(I-K_{\beta}\right)^{-1}$ as a bounded operator on the Banach space $C_{l}^{+}$. (Here, $I$ is the identity operator.)

Theorem 2. (i) Assume $1 \notin \sigma(K)$. Then, for $\beta$ sufficiently large, $\left(I-K_{\beta}\right)^{-1}$ exists as a uniformly bounded linear operator on $C_{l}^{+}$. That is, there exists $M>0$, with $M$ independent of $\beta$, such that $\left\|\left(I-K_{\beta}\right)^{-1}\right\| \leqslant M$, for all $\beta$ sufficiently large.

(ii) Assume also $f \in C_{l}^{+}$. Then, for $\beta$ sufficiently large, $y_{\beta}$ and $z_{\beta}$ exist uniquely in $C_{l}^{+}$, and are bounded uniformly in $\beta$. 
Proof. (i) The result is trivial if $\|K\|<1$, since then $\left\|K_{\beta}\right\| \leqslant\|K\|<1$. For $1 \leqslant\|K\|$, the existence and uniform boundedness of $\left(I-K_{\beta}\right)^{-1}$ as an operator on $X^{+}$, for $\beta$ sufficiently large, has been proved in [1] by a novel application of the Arzelà-Ascoli theorem. (More recently, a constructive proof of this result has been given in [5].) The extension of that result to the space $C_{l}^{+}$is proved in [7].

(ii) On noting that $f_{\beta}$ in (6) is bounded uniformly in $\beta$, these results follow immediately from (i).

To establish the uniform convergence of $z_{\beta}$ to $y$, we write the exact equation (1) as

$$
y-K_{\beta} y=f+\left(K-K_{\beta}\right) y,
$$

and subtract (6) from this equation, obtaining

$$
\left(I-K_{\beta}\right)\left(y-z_{\beta}\right)=r_{\beta} \text {, or } y-z_{\beta}=\left(I-K_{\beta}\right)^{-1} r_{\beta},
$$

where

$$
r_{\beta}(t)=\int_{\beta}^{\infty} k(t, s)[y(s)-y(\infty)] d s
$$

Clearly,

$$
\left\|r_{\beta}\right\| \leqslant\|K\| \sup _{s \geqslant \beta}|y(s)-y(\infty)|,
$$

and so, using the result of Theorem 2 ,

$$
\left\|y-z_{\beta}\right\| \leqslant M\|K\| \sup _{s \geqslant \beta}|y(s)-y(\infty)|,
$$

which converges to zero as $\beta \rightarrow \infty$. Thus we have proved the following theorem:

Theorem 3. Assume $1 \notin \sigma(K)$ and $f \in C_{l}^{+}$. Then for $\beta$ sufficiently large, the error in the modified finite-section approximation $z_{\beta}$ satisfies

$$
\left\|y-z_{\beta}\right\| \leqslant M\|K\| \sup _{s \geqslant \beta}|y(s)-y(\infty)|,
$$

where $M$ is the constant in Theorem 2. Thus, $z_{\beta}$ converges uniformly to y as $\beta \rightarrow \infty$.

If, as will often be the case, $y$ is monotone for sufficiently large argument, then the bound in the theorem becomes simply

$$
\left\|y-z_{\beta}\right\| \leqslant M\|K\||y(\beta)-y(\infty)| .
$$

Thus, the rate at which $z_{\beta}$ converges to $y$ depends on the rate at which $y(\beta)$ approaches $y(\infty)$.

4. Example. In this section we consider an example, similar to Example 1 of [2], on which the performance of the approximations $y_{\beta}$ and $z_{\beta}$ can be compared. The example is

$$
y(t)-\int_{0}^{\infty} \kappa(t-s) y(s) d s=f(t), \quad t \in \mathbf{R}^{+},
$$

with

$$
\kappa(u)=\frac{2}{\lambda \pi}\left(e^{u}+e^{-u}\right)^{-1}
$$

and

$$
f(t)=1-e^{-t}-\frac{2}{\lambda \pi}\left[\tan ^{-1}\left(e^{t}\right)-\frac{1}{2} e^{-t} \log \left(1+e^{2 t}\right)\right]
$$


where $\lambda \neq 0$ is a real number. It can easily be verified that $y(t)=1-e^{-t}$ is an exact solution of this problem for all $\lambda$. A kernel of this type arises in a reformulation, using the double-layer potential, of Laplace's equation on a wedge of angle $\pi / 2$ [3, Eq. (2.11)].

For this example, the norm of the integral operator $K$ is $\|K\|=1 /|\lambda|$. Moreover, by using the results in Section 8 of [1] and Section 6.10 of [8], one can show that the spectrum of $\lambda K$ in the space $X^{+}$(or, equivalently, in the space $C_{l}^{+}$) is $[0,1]$. Since $1 \in \sigma(K)$ if and only if $\lambda \in \sigma(\lambda K)=[0,1]$, it follows that the inverse, $(I-K)^{-1}$, exists as a bounded operator on $C_{l}^{+}$for all $\lambda$ outside the interval $[0,1]$. Finally,

$$
\chi=1 / \lambda, \quad f(\infty)=1-1 / \lambda,
$$

so that Theorem 1 predicts $y(\infty)=1$ for $\lambda \notin[0,1]$, which is consistent with the known exact solution.

The exact solutions of the conventional and modified finite-section equations (5) and (6) are not available for this example, so we have to resort to standard numerical methods to obtain numerical results. Any method could be used, as long as it is of sufficient accuracy. For simplicity we used the quadrature (Nyström) method, based on Simpson's rule, with a step-length $h=0.005$. The resulting approximate solutions of (5) and (6) are denoted below by $Y_{\beta}$ and $Z_{\beta}$, respectively. Numerical experiments suggest that in fact they differ insignificantly from the corresponding exact quantities $y_{\beta}$ and $z_{\beta}$.

First we consider the example with $\lambda=2$. In this case, $\|K\|=\frac{1}{2}$, and since $\left\|K_{\beta}\right\| \leqslant\|K\|$, we have the bound $\left\|\left(I-K_{\beta}\right)^{-1}\right\| \leqslant\left(1-\left\|K_{\beta}\right\|\right)^{-1} \leqslant 2$. Using the fact that $y(t)=1-e^{-t}$, the bound from Theorem 3 ,

$$
\left\|y-z_{\beta}\right\| \leqslant M\|K\| \sup _{s \geqslant \beta}|y(s)-y(\infty)|
$$

now becomes

$$
\left\|y-z_{\beta}\right\| \leqslant e^{-\beta} \text {. }
$$

Numerical results for $\left\|y-Z_{\beta}\right\|$ for four values of $\beta$ are given in Table 1 . In every case the theoretical bound is satisfied comfortably.

TABLE 1

Numerical results for the example with $\lambda=2$, illustrating the validity of the bound in Theorem 3.

\begin{tabular}{|r|c|c|}
\hline$\beta$ & $\left\|y-Z_{\beta}\right\|$ & $\begin{array}{c}\text { bound from } \\
\text { Theorem } 3\left(=e^{-\beta}\right)\end{array}$ \\
\hline 4 & $0.2510^{-2}$ & $0.1810^{-1}$ \\
6 & $0.3310^{-3}$ & $0.2510^{-2}$ \\
8 & $0.4510^{-4}$ & $0.3410^{-3}$ \\
10 & $0.6110^{-5}$ & $0.4510^{-4}$ \\
\hline
\end{tabular}

Next, we consider the same example, with $\lambda=-0.5$. In this case $\|K\|=2$, so that Theorem 3 yields

$$
\left\|y-z_{\beta}\right\| \leqslant 2 M e^{-\beta}
$$

with an unknown value for the constant $M$. The observed behavior of $\left\|y-Z_{\beta}\right\|$ as a function of $\beta$ (not shown) was broadly similar to that in Table 1 . In Figure 1 we 


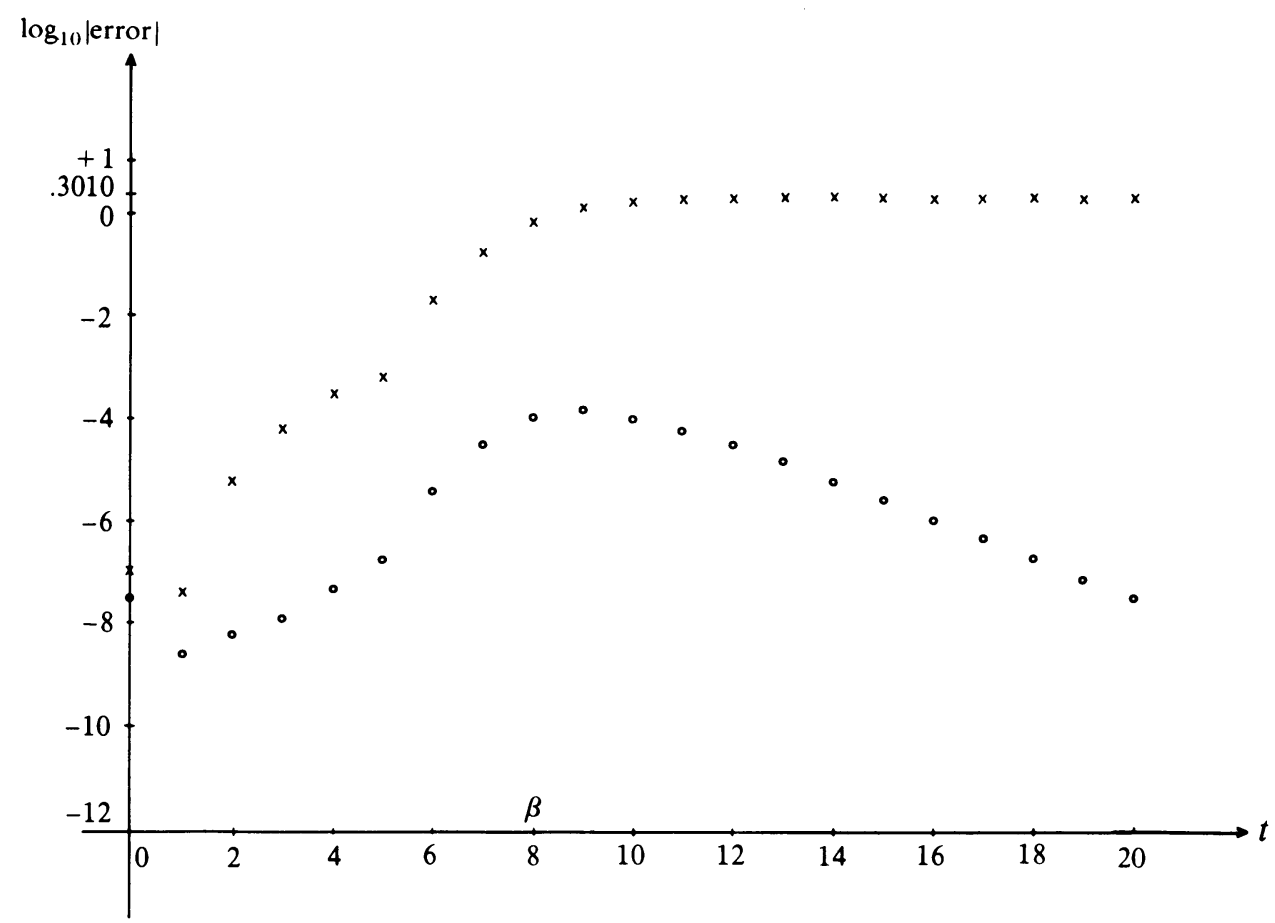

FIGURE 1

Comparison of approximation properties of $Z_{\beta}$ and $Y_{\beta}$ for example with $\lambda=-0.5$ and $\beta=8$. Here $x=\log _{10}\left|y(t)-Y_{\beta}(t)\right|$, and $\circ=\log _{10}\left|y(t)-Z_{\beta}(t)\right|$.

show how the error for $\lambda=-0.5$ behaves as a function of $t$ for a particular value of $\beta$, namely $\beta=8$. Specifically, the plotted quantities are $\log _{10}\left|y(t)-Z_{\beta}(t)\right|$ (shown by circles) and $\log _{10}\left|y(t)-Y_{\beta}(t)\right|$ (shown by crosses). As predicted by Theorem 1 , one observes that $\left|y(t)-Z_{\beta}(t)\right| \rightarrow 0$ as $t \rightarrow \infty$, whereas $\log _{10}\left|y(t)-Y_{\beta}(t)\right|$ converges to 0.301 , corresponding to $\left|y(t)-Y_{\beta}(t)\right| \rightarrow 2$. It is apparent that the modified approximation is already much superior to the conventional finite-section approximation for this value of $\beta$, and the superiority can be expected to increase as $\beta$ increases. The modified approximation, not surprisingly, is seen to have its greatest error in the vicinity of the cut-off parameter $\beta$.

Acknowledgment. I. H. Sloan acknowledges the support of the UK Science and Engineering Research Council.

School of Mathematics

University of New South Wales

Sydney, N. S. W. 2033, Australia

School of Mathematics

University of Bath

Claverton Down

Bath BA2 7AY, Avon, England

1. P. M. Anselone \& I. H. Sloan, "Integral equations on the half-line," J. Integral Equations. (To appear.)

2. K. E. AtKInson, "The numerical solution of integral equations on the half-line," SIAM J. Numer. Anal., v. 6, 1969, pp. 375-397. 
3. K. E. AtKinson \& F. DE Hoog, "The numerical solution of Laplace's equation on a wedge," IMA J. Numer. Anal., v. 4, 1984, pp. 19-41.

4. G. D. FinN \& J. T. JefFeries, "Studies in spectral line formation. I. Formulation and simple applications,” J. Quant. Spectrosc. Radiat. Transfer, v. 8, 1968, pp. 1675-1703.

5. F. DE HOOG \& I. H. SlOAN, "The finite-section approximation for integral equations on the half-line." J. Austral. Math. Soc. Ser. B. (Submitted.)

6. I. H. SLOAN, "Quadrature methods for integral equations of the second kind over infinite intervals," Math. Comp., v. 36, 1981, pp. 511-523.

7. I. H. SloAN \& A. SPENCE, "Projection methods for integral equations on the half-line," IMA $J$. Numer. Anal., v. 6, 1986, pp. 153-172.

8. E. C. Titchmarsh, Introduction to the Theory of Fourier Integrals, Oxford Univ. Press, Oxford, 1937. 